that $p$ is not zero. Let $r=q p$ where $q>1$. By (9) $g_{i+1}=g_{1}+i k$ for $i=1,2, \cdots, q p-1$. Hence $g_{p+1}=g_{1}+p k=g_{1}$, contradicting $g_{1} \neq g_{p+1}$. It follows that the $g$ 's may be grouped into cosets of $K$, whence the theorem is proved.

Corollary 6. Let $S$ be a set of $n$ distinct elements $g_{1}, \cdots, g_{n}$ of an abelian group $G$, where the elements of $S$ are not the elements comprising cosets of a cyclic subgroup of $G$. Let the sum of the elements in $S$ be denoted by $h$. For each element $g$ in $G$ there is a proper representation $\left(g_{1}, \cdots, g_{i-1}, g_{i}+k, g_{i+1}, \cdots, g_{n}\right)$ of $g$ where $k=g-h$.

The Institute for Advanced Study

\title{
THE MAXIMUM NUMBER OF DISTINCT CONTACTS OF TWO ALGEBRAIC SURFACES*
}

\section{T. R. HOLLCROFT}

1. Introduction. For more than half a century, it has been known that the maximum number of distinct contacts of two algebraic surfaces whose intersection curve is irreducible is the genus of that curve. $\dagger$

The number of contacts of two surfaces whose entire intersection curve consists of straight lines has been found. $\ddagger$ This is the maximum number of contacts of two surfaces of given orders.

The purpose of this paper is to obtain the maximum number of distinct contacts of two algebraic surfaces when the in tersection curve consists of any given number of components of given orders and genera. Cases are treated in which the two surfaces have singular points or singular curves in common.

2. Method. From any point of $S_{3}$ not on the developable of $C$, a space curve $C$ with $h$ apparent double points projects into a plane curve $C^{\prime}$ with $h$ nodes. Since $C^{\prime}$ may have $p$ additional nodes, where $p$ is the genus of both $C$ and $C^{\prime}$, the space curve $C$ may have $p$ nodes. The necessary and sufficient condition for the two surfaces to have a contact is for $C$ to have a node. $\S$

If $C$ is irreducible and is the complete intersection of two surfaces $M$ and $N$, the maximum number of contacts of $M$ and $N$ is the genus

* Presented to the Society, December 28, 1937.

$\dagger$ E. Pascal, Repertorium der höheren Mathematik, vol. 2 (1902), p. 225. In a later supplementary volume of the Repertorium (vol. $2_{2}$ (1922), pp. 653-656), more than two pages are devoted to the topic, "Contacts of two surfaces," but the problem of this paper is not treated.

¥ Encyklopädie der mathematischen Wissenschaften, vol. 3, C, 9, (1926), p. 1277.

$\S$ E. Pascal, Repertorium der höheren Mathematik, vol. $2_{2}$ (1922), p. 654. 
of $C$. This is the case referred to in the first paragraph of the preceding section.

If $C$ is reducible, $M$ and $N$ have a contact at each simple intersection of the components of $C$ (which count as nodes of $C$ ) and also at each node of any component. The maximum number of possible contacts is, therefore, the total sum of the genera of all the components and the number of intersections of the components, taken two at a time. This sum is to be found when $C$ consists of any given number of components of given orders and genera, and when the two surfaces have singular points or curves in common.

3. Surfaces with no common singularities. Let $M$ and $N$ be two algebraic surfaces of orders $\mu$ and $\nu$ with no singularities in common. These surfaces intersect in a curve $C$ of order $n=\mu \nu$ which consists of $\alpha$ components $C_{j}$, of orders $n_{j}$, genera $p_{j}$, and with $h_{j}$ apparent double points, respectively.

Any two components $C_{j}$ and $C_{k}$ intersect in $i_{j k}$ points and have $I_{j k}$ apparent intersections with $i_{j k}+I_{j k}=n_{j} n_{k}$. The total number of intersections of the $\alpha$ components of $C$ is $\frac{1}{2} \sum i_{j k},(j \neq k ; j, k=1, \cdots, \alpha)$. A similar expression gives the total number of apparent intersections of all the components, and there results the relation

$$
\sum i_{j k}+\sum I_{j k}=\sum n_{j} n_{k}, \quad j \neq k ; j, k=1, \cdots, \alpha .
$$

The apparent intersections of the components count as apparent double points of the total intersection $C$. This is expressed by the relation

$$
\sum h_{j}+\frac{1}{2} \sum I_{j k}=\frac{1}{2} \mu \nu(\mu-1)(\nu-1) .
$$

The sum of the genera of the components is

$$
\sum p_{j}=\frac{1}{2} \sum\left(n_{j}-1\right)\left(n_{j}-2\right)-\sum h_{j} \text {. }
$$

The maximum number $T$ of distinct contacts of $M$ and $N$ is, therefor, $T=\frac{1}{2} \sum i_{j k}+\sum p_{j}$. Substituting in this expression the value of $\frac{1}{2} \sum i_{j k}$ obtained from (1) and (2) by eliminating $\frac{1}{2} \sum I_{j k}$ and the value of $\sum p_{j}$ given in (3), we obtain

$$
T=\frac{1}{2} \mu \nu(\mu+\nu-4)+\alpha .
$$

This remarkably simple result establishes the following theorem:

The maximum number $T$ of distinct contacts of two algebraic surfaces is independent of the orders and genera of the components of the intersection curve, and, moreover, $T$ is increased by one and only one for each additional component. 
Of the total number $T$ of possible contacts, the surfaces $M$ and $N$ have $\frac{1}{2} \sum i_{j k}$ contacts and may have $\sum p_{j}$ additional contacts. Each of these two numbers is a function of both the orders and genera of the components of $C$, but their sum $T$ is not.

From the expression (4), it appears that the greatest possible number of distinct contacts of two surfaces of orders $\mu$ and $\nu$ occurs when the number of components $\alpha$ is a maximum. The maximum value of $\alpha$ is $\mu \nu$; that is, the intersection consists entirely of lines. The number of contacts in this case is

$$
\frac{1}{2} \mu \nu(\mu+\nu-4)+\mu \nu=\frac{1}{2} \mu \nu(\mu+\nu-2) .^{*}
$$

This maximum number of contacts for two surfaces of given order not only can be, but must be attained when $C$ consists of $\mu \nu$ lines.

The developable surface of $C$ is of order $\mu \nu(\mu+\nu-2)$. When $C$ consists of $\mu \nu$ lines, this surface degenerates into $\frac{1}{2} \mu \nu(\mu+\nu-2)$ distinct planes, each counted twice. Each of these planes is determined by a pair of intersecting lines of the $\mu \nu$ lines common to $M$ and $N$. Each of the $\frac{1}{2} \mu \nu(\mu+\nu-2)$ planes is, therefore, tangent to both $M$ and $N$ at the intersection of the line pair determining that plane.

4. Application to quadrics. If $M$ and $N$ are quadrics, $\mu=\nu=2$ and $T=\alpha$; that is, the number of possible contacts is always equal to the number of components of the intersection curve.

The only pairs of proper quadrics in $S_{3}$ that do not have contact with each other are those whose intersection is an elliptic quartic. Also, all pairs of proper quadrics, whose intersection consists of $1 \leqq \alpha \leqq 4$ distinct rational components, have $\alpha$ contacts. If $\alpha \geqq 2$, all components must be rational.

The maximum number of distinct contacts of two quadrics is four, which occurs when the quadrics intersect in four lines. In this case, the equations of $M$ and $N$ may be written $\dagger$

$$
\begin{aligned}
& M: \lambda_{1}\left(x_{1}^{2}+x_{2}^{2}\right)+\lambda_{2}\left(x_{3}^{2}+x_{4}^{2}\right)=0, \\
& N: x_{1}^{2}+x_{2}^{2}+x_{3}^{2}+x_{4}^{2}=0 .
\end{aligned}
$$

The equation of the developable surface of the complete intersection of these two quadrics is found to be

$$
\left(x_{1}^{2}+x_{2}^{2}\right)\left(x_{3}^{2}+x_{4}^{2}\right)=0 .
$$

\footnotetext{
* Encyklopädie, loc. cit. This expression may be derived directly. A set of $\mu \nu$ coplanar lines has $\frac{1}{2} \mu \nu(\mu \nu-1)$ intersections. When the $\mu \nu$ lines constitute the total intersection of $M$ and $N, h=\frac{1}{2} \mu \nu(\mu-1)(\nu-1)$ intersections are apparent, and the remaining $\frac{1}{2} \mu \nu(\mu \nu-1)-h=\frac{1}{2} \mu \nu(\mu+\nu-2)$ are actual intersections of the $\mu \nu$ lines.

$\dagger$ V. Snyder and C. H. Sisam, Analytical Geometry of Space, 1917, p. 163.
} 
This surface consists of four planes, each of which passes through a pair of lines common to $M$ and $N$.

5. Surfaces with singular points in common. Let $M$ and $N$ have in common $\beta$ points $P_{i}$ which are $r_{i}$-fold on $M$ and $s_{i}$-fold on $N$. Three cases will be considered.

CAsE 1 . None of the $\beta$ points $P_{i}$ lie at an intersection of the $\alpha$ components of $C$.

The complete intersection curve $C$ has

$$
h=\frac{1}{2} \mu \nu(\mu-1)(\nu-1)-\frac{1}{2} \sum r_{i} s_{i}\left(r_{i}-1\right)\left(s_{i}-1\right), \quad i=1, \cdots, \beta .^{*}
$$

This reduction in $h$ applies only to bisecants of the individual components and not to those of pairs of components.

The surface $C$ has $\beta$ multiple points of orders $r_{i} s_{i}$ which occur at distinct points on components of $C$. Hence the sum $\sum^{\alpha} p_{j}$ of the genera of the components $C_{j}$ is reduced by

$$
\begin{array}{r}
\frac{1}{2} \sum r_{i} s_{i}\left(r_{i} s_{i}-1\right)-\frac{1}{2} \sum r_{i} s_{i}\left(r_{i}-1\right)\left(s_{i}-1\right)=\frac{1}{2} \sum r_{i} s_{i}\left(r_{i}+s_{i}-2\right), \\
i=1, \cdots, \beta .
\end{array}
$$

Substituting this reduction in (4), we obtain

$$
T=\frac{1}{2} \mu \nu(\mu+\nu-4)+\alpha-\frac{1}{2} \sum^{\beta} r_{i} s_{i}\left(r_{i}+s_{i}-2\right) .
$$

The number of intersections $\frac{1}{2} \sum i_{j k},(j \neq k ; j, k=1, \cdots, \alpha)$, is not affected by the $\beta$ multiple points in this case.

There is no relation between $\alpha$ and $\beta$. Two or more multiple points may be on one component, so that $\beta$ may be larger than $\alpha$. The only restriction on the multiple points is that their size and number must not be so great as to cause any component to break up into curves of lower order intersecting at certain of the points $P_{i}$.

CASE 2. The $\beta$ multiple points $P_{i}$ which are $r_{i}$-fold on $M$ and $s_{i}$-fold on $N$ are so situated that two and only two component curves $C_{j}$ and $C_{k}$ of $C$ pass through each $P_{i}$. The composite multiplicity of $P_{i}$ on $C_{j}$ and $C_{k}$ is $r_{i} s_{i}$. Let the multiplicity of $P_{i}$ on $C_{j}$ be $\rho_{j}$ and on $C_{k}, \rho_{k}$. Then

$$
\rho_{j}+\rho_{k}=r_{i} s_{i} .
$$

The components $C_{j}$ and $C_{k}$ have $\rho_{j} \rho_{k}$ intersections with each other at $P_{i}$ in addition to $\frac{1}{2} \rho_{j}\left(\rho_{j}-1\right)$ intersections of branches of $C_{j}$ and $\frac{1}{2} \rho_{k}\left(\rho_{k}-1\right)$ intersections of branches of $C_{k}$, that is,

* N. Salvatore-Dino, Sul genere delle curve gobbe, Rendiconti di Napoli, vol. 18 (1879), pp. 132-136. 


$$
\rho_{j} \rho_{k}+\frac{1}{2} \rho_{j}\left(\rho_{j}-1\right)+\frac{1}{2} \rho_{k}\left(\rho_{k}-1\right)=\frac{1}{2} r_{i} s_{i}\left(r_{i} s_{i}-1\right) .
$$

The $\rho_{j} \rho_{k}$ intersections of $C_{j}$ and $C_{k}$ at $P_{i}$ count among the $i_{j k}$ intersections of $C_{j}$ and $C_{k}$, but since these $\rho_{j} \rho_{k}$ intersections lie at $P_{i}$, they are associated with no contacts of $M$ and $N$. The number of contacts of $M$ and $N$ that would occur at intersections of $C_{j}$ and $C_{k}$ is, therefore, reduced by $\rho_{j} \rho_{k}$.

The genus $p_{j}$ of $C_{j}$ is

$$
p_{j}=\frac{1}{2}\left(n_{j}-1\right)\left(n_{j}-2\right)-h_{j}-\frac{1}{2} \rho_{j}\left(\rho_{j}-1\right),
$$

and similarly for the genus $p_{k}$ of $C_{k}$. The genera of component curves that do not pass through points $P_{\boldsymbol{i}}$ are not affected. Since there are $\beta$ points $P_{i}$, it follows that $\beta$ curves $C_{j}$ and $\beta$ curves $C_{k}$ are affected. Hence,

$$
\begin{aligned}
T= & \frac{1}{2} \mu \nu(\mu+\nu-4)+\alpha-\frac{1}{2} \sum^{\beta} \rho_{j}\left(\rho_{j}-1\right) \\
& -\frac{1}{2} \sum^{\beta} \rho_{k}\left(\rho_{k}-1\right)-\frac{1}{2} \sum^{\beta} \rho_{j} \rho_{k},
\end{aligned}
$$$$
j \neq k,
$$

which reduces to

$$
T=\frac{1}{2} \mu \nu(\mu+\nu-4)+\alpha-\frac{1}{2} \sum^{\beta} r_{i} s_{i}\left(r_{i} s_{i}-1\right) .
$$

It has been assumed that a given component $C_{j}$ may have a point $P_{i}$ at its intersection with one component $C_{k}$, but not another multiple point at its intersection with a third component. This restriction is not necessary, however, since the reduction in the $\operatorname{sum} \sum p_{j}+\frac{1}{2} \sum i_{j k}$ is the same whether two points $P_{i}$ lie on two distinct pairs of components, or at two of the intersections of one component with two others, or at two intersections of the same two components. The same argument holds for three or more points $P_{i}$.

CASE 3 . In this case, $\beta_{1}$ points $P_{i}$ satisfy the conditions of Case 1 and $\beta_{2}$ of Case 2. The resulting value of $T$ is a combination of formulas (5) and (6):

$$
T=\frac{1}{2} \mu \nu(\mu+\nu-4)+\alpha-\frac{1}{2} \sum^{\beta_{1}} r_{i} s_{i}\left(r_{i}+s_{i}-2\right)-\frac{1}{2} \sum^{\beta_{2}} r_{i} s_{i}\left(r_{i} s_{i}-1\right) .
$$

6. Surfaces with a common contact curve. If $M$ and $N$ have simple contact along a curve $C_{1}$, then at nodes of $C_{1}$ or at intersections of the other components of $f$ with $C_{1}, M$ and $N$ have contact of higher order than at ordinary points of $C_{1}$. Since all such contacts occur on $C_{1}$, they are not distinct contacts, and will not be counted in the maximum number of distinct contacts.

If $C$ consists of $\alpha$ components $C_{j},(j=1, \cdots, \alpha)$, of which $\alpha_{1}$ com- 
ponents $C_{1}, \cdots, C_{\alpha_{1}}$ are contact curves, the maximum number of distinct contacts of $M$ and $N$, that is, the maximum number $T$ of contacts at points not on any of the contact components, is $T=\sum p_{j}+\frac{1}{2} \sum i_{j k},\left(j \neq k ; j, k=\alpha_{1}+1, \alpha_{1}+2, \cdots, \alpha\right)$. This does not reduce to a function of $\mu, \nu$, and $\alpha$ as is the case when all of the components are simple.

7. Surfaces with a common multiple curve. Let the total intersection of $M$ and $N$ consist of two components $C_{1}$ and $C_{2}$ of orders $n_{1}, n_{2}$ and genera $p_{1}, p_{2}$, such that $C_{1}$ is $i_{1}$-fold on $M$ and $i_{2}$-fold on $N$. The contacts of $M$ and $N$ at nodes of $C_{1}$ and $C_{2}$ and at intersections of $C_{1}$ and $C_{2}$ will be distinct contacts as before. If $t$ is the number of intersections of $C_{1}$ and $C_{2}$, the maximum number of distinct contacts of $M$ and $N$ is $p_{1}+p_{2}+t$.

The value of this expression in terms of $\mu, \nu, i_{1}, i_{2}, n_{1}, p_{1}$ is obtained by using formulas (A) and (3) of a former paper.*

More generally, if $M$ and $N$ intersect in $\alpha$ components, $C_{j}$ of orders $n_{j}$ and genera $p_{j}$, and if one component $C_{1}$ is $i_{1}$-fold on $M$ and $i_{2}$-fold on $N$ and the remaining components are simple on both surfaces, the maximum number of contacts of $M$ and $N$ is

$$
\begin{aligned}
\frac{1}{2}\left(i_{1}+i_{2}-3\right)\left[2 i _ { 1 } i _ { 2 } \left(2 n_{1}+\right.\right. & \left.\left.p_{1}-1\right)-n_{1}\left(i_{2} \mu+i_{1} \nu\right)\right] \\
& +\left(\mu \nu-i_{1} i_{2} n_{1}\right)(\mu+\nu-4)+p_{1}+\alpha-1 .
\end{aligned}
$$

Thus, in this case, the maximum number of contacts of $M$ and $N$ can be obtained when the orders of the two surfaces, the number of components, and the order, genus, and multiplicities of the multiple curve are known.

8. Stationary contacts of two surfaces. The maximum number of distinct stationary contacts of two surfaces can be obtained by similar methods when the problem of finding the maximum number of cusps of a plane curve of given order and genus has been solved.

Wells College

* T. R. Hollcroft, Characteristics of multiple curves and their residuals, this Bulletin, vol. 39 (1933), pp. 960-961. 